\title{
Optimizing Maximum Ejecting Speed for a Gaussian Accelerator Cannon
}

AUTHORS: Mohammad Reza Noormandipour*, Seyed Ali Reza Manavi, Mahdi Sarikhani, Elmira Vahidi

SCHOOL: Department of Physics, Shahid Beheshti University, Velenjak, Tehran, Iran

\section{Abstract}

This paper aims to find the maximum speed of an ejected steel ball from a magnetic cannon and determine its relation to other setup parameters. A magnet induces dipole moments in steel balls inside a magnetic cannon and makes them stick together along a straight line. At the moment of collision, this attraction reduces the motion of the balls in other directions. This creates a more efficient momentum transfer along the series of steel balls and consequently, a higher speed for the ejected ball. The striking ball was released from a height of $6 \mathrm{~cm}$ above the rail and collided with two magnets, followed by three balls. The speed was calculated using the projectile range formula. The speed was found to be a damping non-monotonic function of the number of balls after the magnet. The effective parameters were studied and the maximum velocity obtained under experimental conditions was $295 \pm 5 \mathrm{~cm} / \mathrm{s}$. Figures were made using SolidWorks software and data were analyzed using Microsoft Excel 2016 and Gnuplot software.

\section{Key Words}

Gauss rifle, neodymium magnets, steel balls

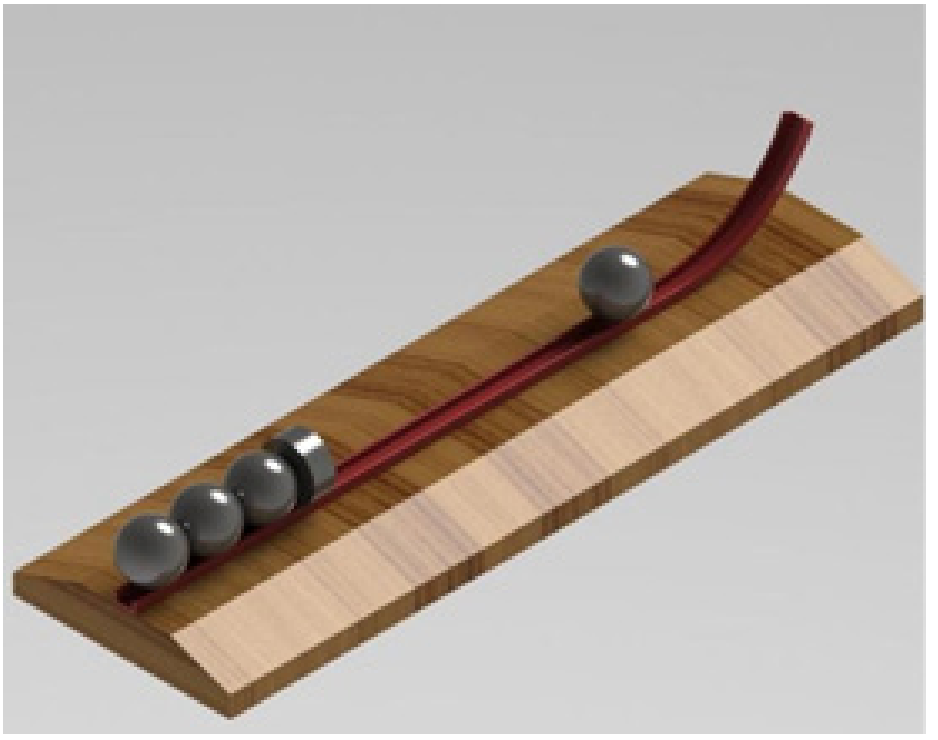

a)

\section{Introduction}

The magnetic cannon, also known as a Gauss rifle, is a series of steel balls connected to a strong magnet. The balls and magnets lie on a non-magnetic rail. Another ball, with a known initial momentum, collides with the sequence of balls and magnets, causing the last ball to eject with a high velocity. This paper aims to determine the conditions under which the final speed of the ejecting ball is maximized. When experimenting with magnetic cannons, it is important to ensure that the magnets are fixed in place to avoid any backward movement. Balls should be aligned along a straight line for optimized momentum transfer. It is preferable for better alignment to use magnets and balls with the same diameter.

When the striking ball approaches the magnet, it accelerates, due to its attraction to the magnet, and collides with the sequence of objects in high velocity (Fig.1). Although the other balls are also attracted to the magnet, this force decays exponentially with increasing distance from the magnet, so the last ball is easily expelled. The magnet induces dipole moments in the balls, causing them to stick together. Due to the increase of friction between the balls, they will not slide over each other, thus reducing the vibrations caused by collision and thereby decreasing the energy loss during momentum transfer. The non-conservative friction force is perpendicular to the direction of momentum transfer, so it is irrelevant to the system in question.

Deriving the relation of the magnetic force between the magnet and the steel balls to the ejection speed proved somewhat complicated. For instance, induced dipole moments in the second ball will induce dipoles in the first ball in the opposite orientation of the di

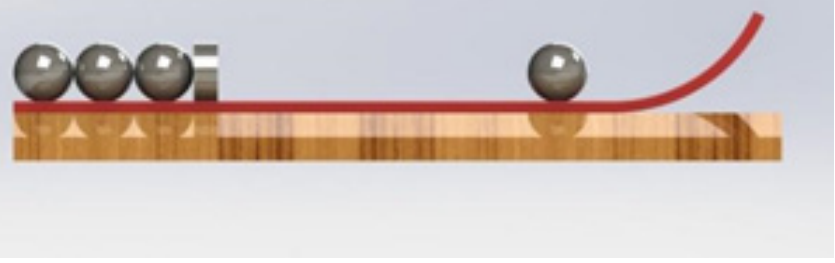

b)

Figure 1: Gauss rifle 
poles caused by the magnet. The same effect happens between the rest of the steel balls. These contributions must be summed and taken into consideration. Additionally, induction of dipoles also depends on the shape of the balls as well as the purity of their alloy.

Effective parameters in relation to the final speed of the cannon were:

1. Initial Velocity

2. Number of balls after the magnet

3. Strength of magnet

4. Number of balls before the magnet

5. Mass of each ball

\section{Materials and Methods}

Setup was constructed using a plastic rail and steel balls with diameter $25 \mathrm{~mm}$ and mass of 80.5 grams. The magnets were grade 42. The grade of a magnet represents its strongest point in the demagnetization curve and is a measure of its strength. The magnets were cylindrical with a diameter $25 \mathrm{~mm}$ and height $10 \mathrm{~mm}$ and were made of an alloy of neodymium, iron, and boron of chemical formula $\mathrm{Nd}_{2} \mathrm{Fe}_{14} \mathrm{~B}$.

At first, the relation of magnetic force with distance between the centers of a ball and magnet was found using the setup installed in Fig.2.
According to relevant literature (1), to create a higher ejecting speed, it is better to place the magnet at beginning of the series of balls so that the striking ball directly collides with it.

The final velocity was measured using the projectile range formula, setting $R$ as range and $h$ as height of the cannon from ground level, as seen in Figure 3. From introductory physics, we know that for a horizontal projectile with a small initial velocity, we can neglect the effect of air resistance and thus the total time before collision with ground is equal to $t=\frac{R}{v}$

Putting this into the equation of free fall, $h=\frac{1}{2} g t^{2}$,

and solvina for $\mathrm{v}$, we can find the magnitude of ejecting velocity as $v=R\left(\frac{g}{2 h}\right)^{\frac{1}{2}} \quad$, where $\mathrm{g}$ is the gravitational constant.

The range formula was used instead of electric speed sensors, because in order to use electric speed sensors, the ejecting ball would have had to travel a horizontal distance over the rail, resulting in energy loss, which would ultimately result in error.

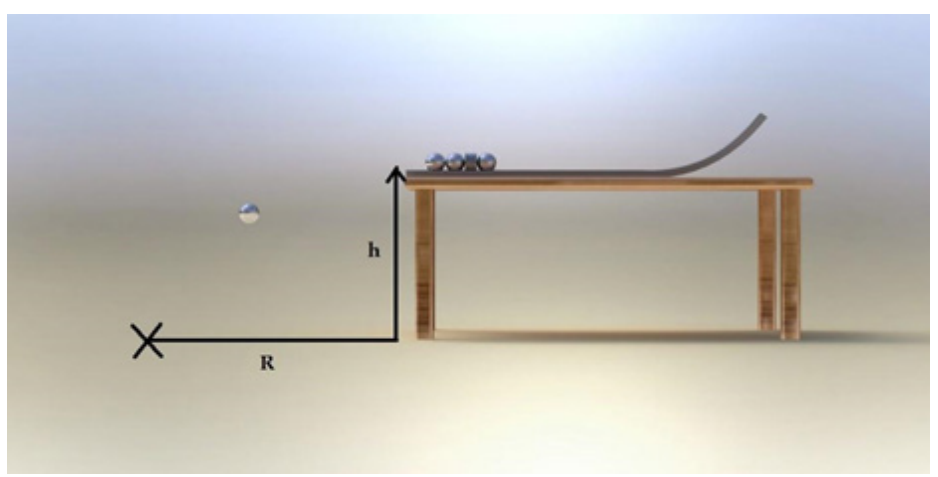

Figure 3: Setup for calculation of ejecting speed using the range and height of projectile

Figure 2: Setup for measuring the force exerted on steel ball due to magnet 


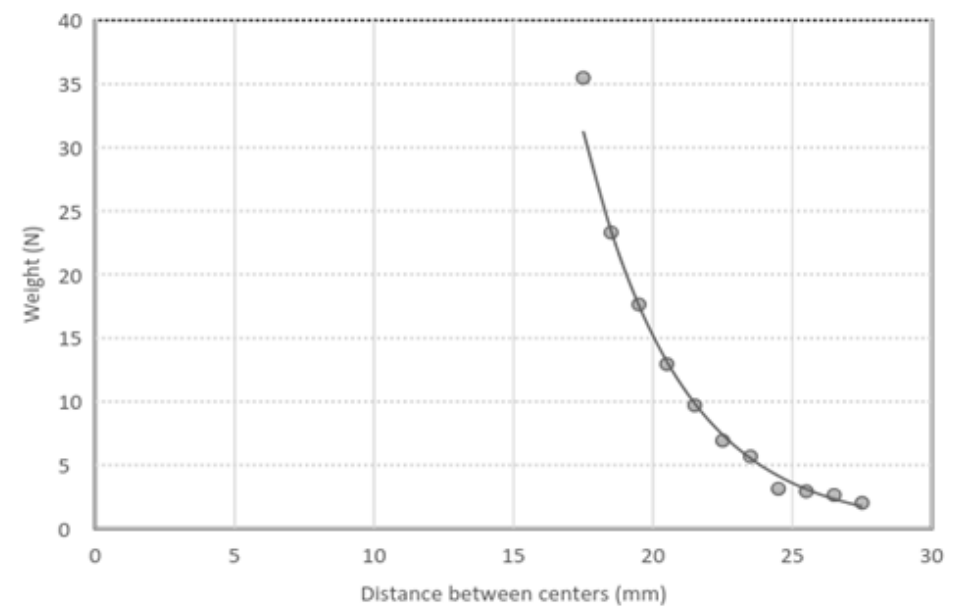

Figure 4: Setup Relation of tolerated weight by magnets with distance between centers of one magnet and one steel ball using setup in Fig.2.

Errors were too small to show in the diagram.

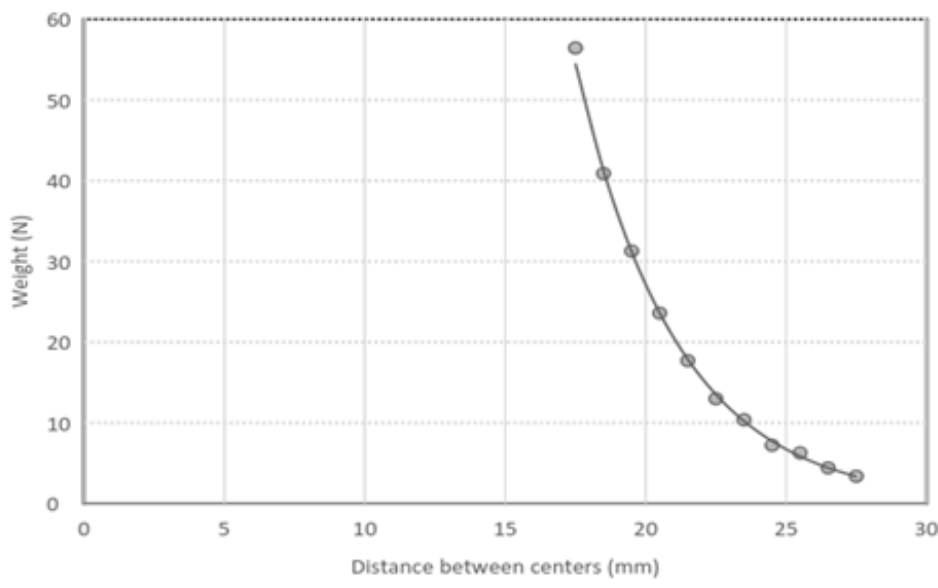

Figure 5: Relation of tolerated weight with distance between centers of two identical magnets and one steel ball using setup in Fig.2.

Errors were too small to show in the diagram.

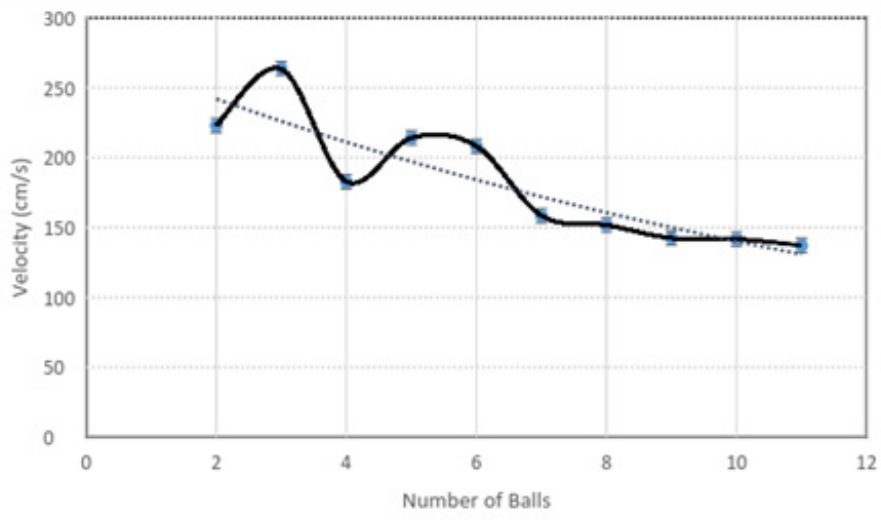

Figure 6: Velocity of the ejected ball vs. the number of balls after one magnet.

\section{Results}

The results of magnetic force for one and two magnets are plotted in figures [4] and [5] respectively.

Then, using a known initial velocity, which was fixed by releasing the striking ball from a constant height and changing the number of balls after the magnet, Figure [6] was plotted.

The maximum final velocity obtained was at three balls after the magnet. The same experiment was performed for two magnets, instead of one, to check the effect of magnet strength. The results are shown in Figure [7]. The maximum velocity with one magnet was $263 \pm 5 \mathrm{~cm} / \mathrm{s}$, and with two magnets was equal to $295 \pm 5 \mathrm{~cm} / \mathrm{s}$.

\section{Discussion}

The results is shown in Diagram [3], the final velocity is a damping non-monotonic function of the number of balls after the magnet. Its base line decays due to energy loss, because of the fact that the induced dipole moment for balls far away from the magnet tends to zero and momentum transfer becomes inefficient due to the vibrations of balls.

Results show that the maximum final velocity occurs when the number of balls after the magnet is equal to three. The same result was also obtained for three identical magnets. Initial velocity needs to be taken into consideration. $V$ is the velocity of the striking ball before collision and $\mathrm{X}$ is its position away from the magnet. A V-X diagram can be plotted using the equation of magnetic force from Figures [4] and [5]. Assuming that the striking ball will not slide over the rail due to friction, the relationship between $\mathrm{V}$ and $\mathrm{X}$ can be determined mathematically.

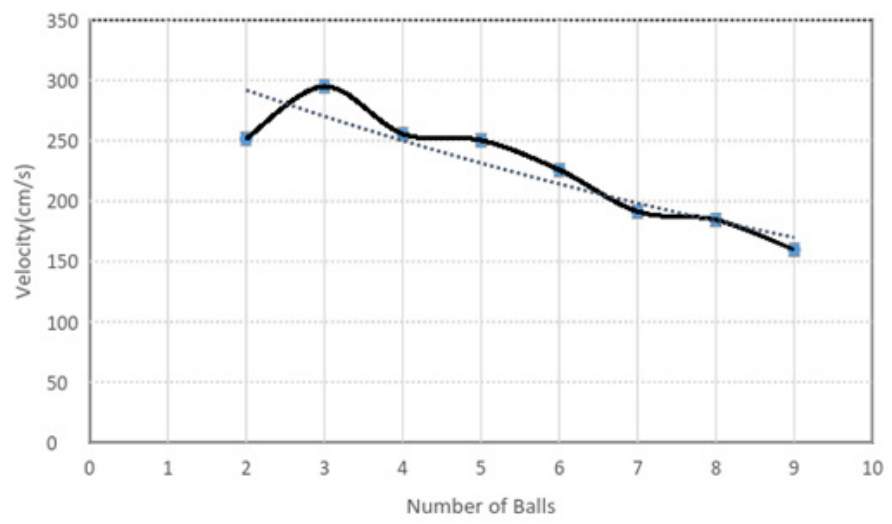

Figure 7: Velocity of ejecting ball vs. number of balls after two identical magnets. 


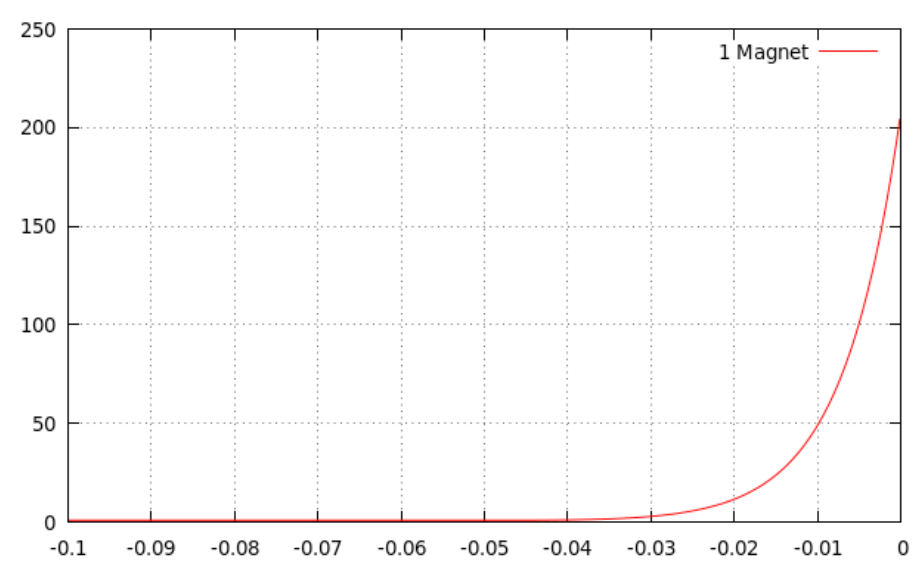

Figure 8: Speed of the striking ball vs. its position before one magnet.

From conservation of energy:

$$
\begin{aligned}
& \frac{1}{2} m v_{i}^{2}+\frac{1}{2} I \omega^{2}=m g h \\
& I_{\text {sphere }}=\frac{2}{5} m R^{2} \quad \& \quad v_{i}=R \omega
\end{aligned}
$$

Sub $I_{\text {sphere }}$ and $v_{i}$ into [1]:

$$
v_{i}^{2}=\frac{10}{7} g h
$$

Through integration:

$$
v=\left[\frac{-2}{m} \int_{x}^{l} F(x) \cdot d x+\frac{10}{7} g h\right]^{\frac{1}{2}}
$$

Numerically integrating and plotting the above function for $\mathrm{V}$ leads to figures [8] and [9] for one and two magnets respectively, with the magnet at position $\mathrm{x}=0$.

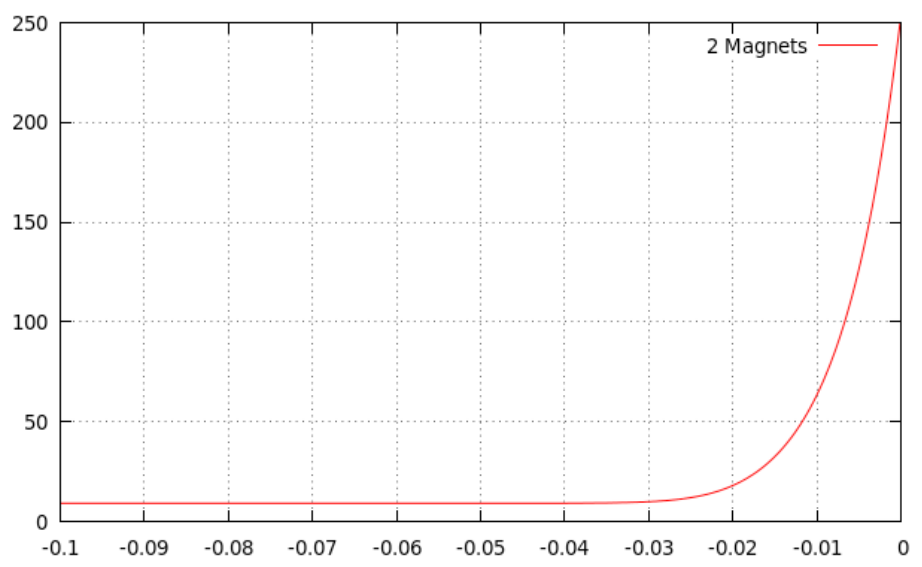

Figure 9: Speed of the striking ball vs. its position before two magnets.

The velocity of the striking ball increases by approximately a factor of 15 in its final $2 \mathrm{~mm}$ to the magnets. This device is called a magnetic accelerator and one may use such multistage accelerations in order to reach a higher final velocity, where in the first stage, the striking ball collides with the first magnet, turning the ejecting ball into the new striking ball for the second stage, which collides with the second magnet, and so on, such that in the ' $n$ 'th stage the final velocity is $v_{f}=v_{0} \sqrt{n}$

\section{Conclusion}

The maximum velocity obtained was $295 \pm 5 \mathrm{~cm} / \mathrm{s}$, when the striking ball was released from a height of $6 \mathrm{~cm}$ above the rail and collided with two magnets followed by three balls.

The reason for the non-monotonic velocity relative to the number of balls that was found is unknown, though is not due to experimental error as the differences between velocities are larger than the error bars, as shown in Figure [6]. Additionally, every value of velocity for the proportional number of balls was determined through repeated experiments.

\section{References}

1. Kirk T. A Magnetic Linear Accelerator. Joseph Henry Laboratories, Princeton University, Princeton, NJ 08544.2003 March.

2. Ucke C, Joachim Schlichting H. Die Magnetkanone. Physik in unserer Zeit, March, 2009;40(1):52-54.

3. Zargham S, Ghaednia H. The Electro Magnetic Cannon, 2010. IYPT Proceedings book. 


\section{REVIEW}

\section{by Dr. Andrew Sazonov,}

Inputmax Ltd.

In this article the authors study an interesting phenomenon that is known as Gauss Rifle or Magnetic Linear Accelerator (http://miniscience.com/projects/Gauss_Rifle/index.html). The theoretical study of the simplest construction of such accelerator (a magnet and two steel marbles) was conducted in [1]. The primary task of this paper is to determine how the effectiveness of the "accelerating element" depends on the number of balls. The system under review is a fascinating object to study: when the "accelerating element" consists of only one ball (magnet plus one ball, bonded to the magnet) - the effect disappears, as the whole system in its initial and final stages has the same magnetic energy. But if a number of balls used is greater than 1, the internal energy of "accelerating element" before and after the impact differs. This allows for a direct conversion of the magnetic energy into a kinetic energy of the ball. Besides, the mechanical energy of the last ball should increase with the number of balls used. This would lead to the increased efficiency of the "accelerating element". On the other hand, the friction losses would increase with the number of balls used. As a result, one should expect the existence of the maximum on the efficiency graph. However, the authors obtained a very interesting result: the effectiveness is oscillating with maximums at odd numbers of balls. It would be interesting to understand the nature of such phenomenon.

This paper could benefit from a more detailed description of the experiment, the description of the method that was used to measure the shooting ball velocity and the pictures with the explanation of the used parameters. Besides the experiment could be simplified by using suspended balls as it would reduce friction on plastic rails and would avoid rotation of the balls that complicates the calculation of the shooting ball velocity. Never the less it is a good paper that provided the reader with an understanding of the phenomenon. 